\title{
Slow Alpha Variant: A Report of a 3 for 1 Ratio Subharmonic
}

\author{
Philippe Beauchemin, Martin Savard
}

Can. J. Neurol. Sci. 2012; 39: 97-98

As a group, unusual electroencephalogram (EEG) patterns are often seen and make interpretation more difficult. Some are not understood on the basis of physiology and clinical implication. For others, no pathological substrate or epileptic condition explains these patterns.

Background activity refers to the EEG rhythm corresponding to the patient's age and state of consciousness. The alpha rhythm is in the range of 8 to $12 \mathrm{Hertz}(\mathrm{Hz})$, has a sinusoidal waveform, blocks with eye opening and alertness and is usually pronounced in posterior regions ${ }^{1}$.

In 1947, Goodwin first called attention to a slow alpha variant rhythm, which predominates in the occipital and adjacent posterior areas ${ }^{2}$. Ten percent of normal subjects' EEG contained alpha variant rhythm. Another source reported it in $0.8 \%$ of asymptomatic people ${ }^{3}$. When it has once been observed, it may be expected to reappear more or less regularly in EEG recordings.

Alpha variant can be fast, corresponding to twice the usual alpha frequency, or slow. The slow alpha variant has a frequency half of the alpha rhythm, usually in the range of 4-5 Hz. It is the definition of a harmonic pattern ${ }^{4}$. It could be, but not invariably, synchronous and symmetrical. The notched appearance waveforms can be more or less continuous while alternating with the regular alpha activity, or even paroxysmal. They often show a strong blocking to eyes opening and, as a rule to photic stimulation, being replaced by a driving effect. It is thought to represent a physiological variation of the basic cortical rhythm.

According to Goodwin, in a few cases, the alpha variant rhythm ratio was 3 to 1 and on rare occasions 4 to 1 compared to the basic rhythm ${ }^{2}$. However, he did not provide any EEG sample and that has never been reported thereafter to our knowledge. We therefore present the first EEG sample of a rhythm showing the usual pattern of the slow alpha variant, but with a rhythm ratio of 1 to 3 .

\section{CASE Report}

Our patient is a 61-year-old woman with first seizure, at age 35 , described as a loss of consciousness with two minutes of generalized tonic-clonic movements. Brain tomography and EEG were normal. Tegretol was started to be taken on a regular basis. The patient never complained of any other seizure. At age 52 and later at age 61, she sought neurological consultation for episodic headaches associated with right peri-orbital and upper lip contractions happening in stressful situations. Brain magnetic resonance imaging (MRI) and carotid ultrasonography were normal.

Using standardized 10-20 international system, two EEG's showed rhythmic 2.5 to $3 \mathrm{~Hz}$ notched slowing in both posterior regions, from occipital to temporal posterior regions (Figure A). Their amplitudes were more pronounced than the normal $9 \mathrm{~Hz}$ background. These bursts of monomorphic slow waves could be either on the left side, on the right side or both, and either synchronous or asynchronous. They occurred randomly each minute and lasted a maximum of six seconds. They never happened during eye opening. They disappeared at eye opening (Figure B) and, at times, appeared at eye closure (Figure C). On first EEG, head movements were suspected. None were seen by the technologist during bursts on the second. No clinical manifestations or changes in vigilance were seen. Hyperventilation slightly increased this intermittent finding and $3-\mathrm{Hz}$ photic stimulation brought a time-locked train of these slow waves bilaterally (Figure D).

\section{DISCUSSION}

This EEG demonstrates the three cardinal features of alpha rhythm, but in paroxysmal $3 \mathrm{~Hz}$ trains of notched waves (Figure A). It represents a third of the $9-\mathrm{Hz}$ alpha rhythm of the patient. This pattern has the following characteristics: 1-the posterior distribution, 2-the reactivity to eye opening (Figure B and C) and to alertness and 3-the reactivity to photic stimulation (Figure D). Moreover, 4-it happens in short bursts of one to six seconds, many times during EEG and, 5-at the frequency of a third of the alpha rhythm. Given these features, it represents a 3 to 1 ratio slow alpha variant, only suggested in the original article from Goodwin $^{2}$. It is interesting to compare this rhythm to other unusual patterns seen in the posterior head regions.

Posterior rhythmic slow wave activity, or PRS, can occur transiently after eye closure ${ }^{4}$. 1-This activity is present in posterior regions, 2-attenuates with eye opening, but 3-does not show any change with other reactivity maneuver. 4-It may also fatigue with repeated testing and 5-it has no relation with the background rhythm. It does not correspond to our patient's EEG.

Some patients with absence seizures have trains of rhythmic slow-wave activity over the posterior regions during interictal periods. 1-Predominantly over the occipital head regions in children aged six to ten years old, 2-this activity attenuates with eye opening. 3-It consists of bilaterally synchronous sinusoidal or fusiform $3 \mathrm{~Hz}$ rhythmic slow waves ${ }^{4}$. Our patient is not in this age group and never experienced absence seizures.

From the Sciences Neurologiques Department, Hôpital de l'Enfant-Jésus, Québec, QC, Canada.

Received May 18, 2011. Final Revisions Submitted July 14, 2011. Correspondence to: Martin Savard, Sciences Neurologiques Department, Hôpital de l'Enfant-Jésus, 1401, 18e Rue, Québec, QC, G1J 1Z4, Canada. 

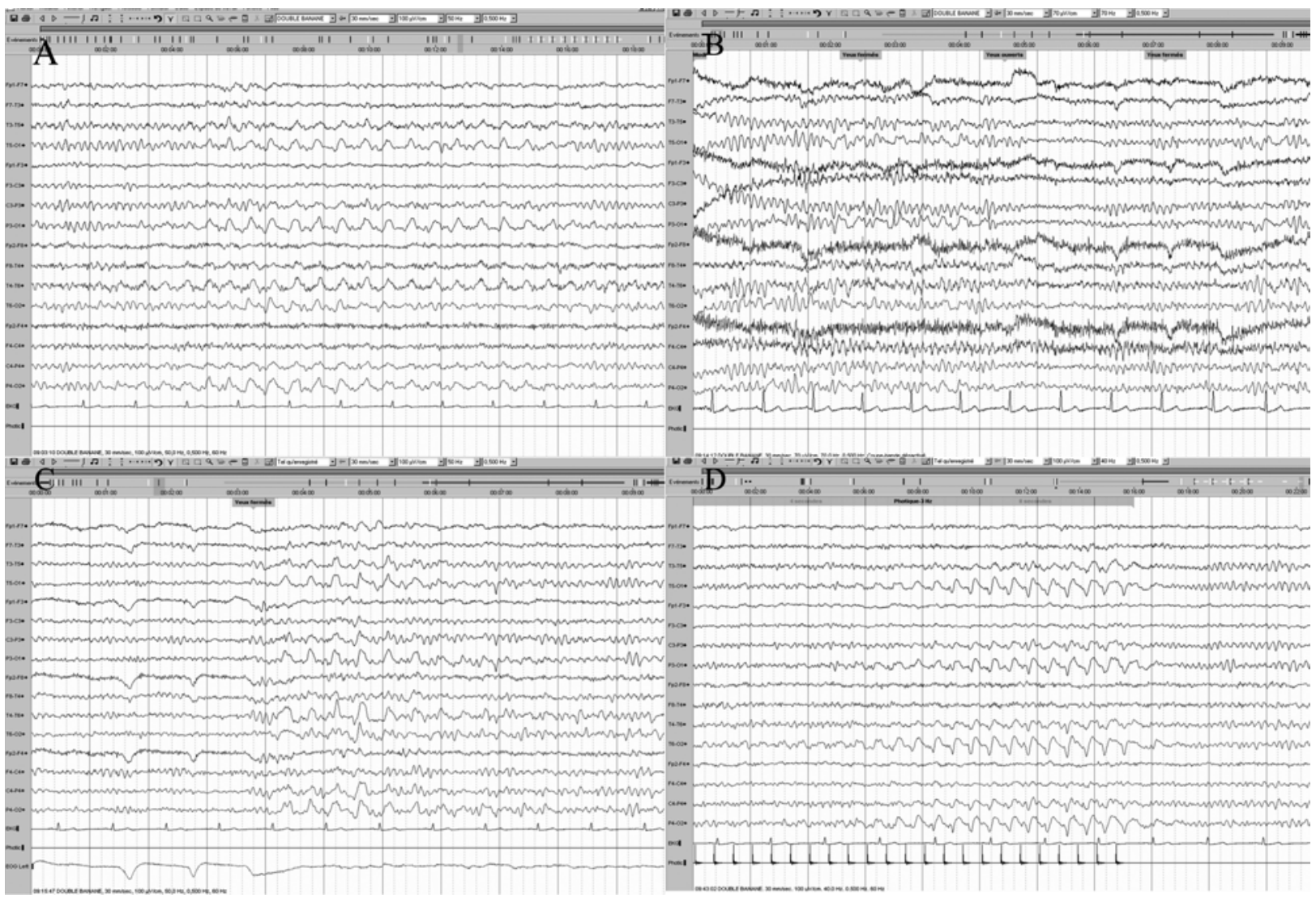

Figure: EEG, with bipolar longitudinal montage, showing trains of bilateral posterior notched slow waves at a 2.5-3 $\mathrm{Hz}$ (A) intermixed with a $9 \mathrm{~Hz}$, background rhythm. It disappeared $(B)$ and reappeared $(C)$ respectively with eye opening and closure and it started during 3-Hz photic stimulation $(D)$.

Subclinical Rhythmic Electrographic Discharges of Adults (SREDA) resembles a seizure pattern on $E^{2} G^{5}$. 1-They are present over both hemispheres and is distributed widely. 2Moreover, they are not known to share reactivity to eye closure and 3-to photic stimuli. 4-They may persist up to several minutes. 5-They are not in a relationship with the normal background rhythm. It happens usually in patients without any history of clinical seizures. Thus, for many reasons, this does not correspond to our case.

Occipital Intermittent Rhythmic Delta Activity (OIRDA) is observed in children from the age 6 to 14 , although it might persist into adolescence and young adult life. 1-As the name implies, this activity happens in the occipital regions. 2-It is blocked by opening of the eyes. 5-It runs in the delta range, but without any relation to the underlying alpha rhythm. It does not have a notched appearance. This one is also improbable.

Repetitive EEG discharges with a relatively abrupt onset and termination and a characteristic pattern of evolution in rhythm and amplitude are generally considered to represent EEG seizure discharges ${ }^{1}$. 1-This ictal activity may happen in any cortical region and spread to the adjacent regions, 2-does not show any reactivity to eye opening, or 3-other reactivity maneuvers. 4Bursts have different lengths and 5-are usually not in any relation to the normal rhythm.

\section{CONCLUSION}

We report a case of a 3 for 1 slow alpha variant. This rare finding has been described in the initial paper on the slow alpha variant, but in our opinion it is not well recognized and it was never published with images before. The pattern rarity makes any discussion about its significance speculative, but its relationship to normal pattern makes us believe it could be a benign variant.

\section{REFERENCES}

1. Noachtar S, Binnie C, Ebersole J, et al. A glossary of terms most commonly used by clinical electroencephalographers and proposal for the report form for the EEG findings. The International Federation of Clinical Neurophysiology. Electroencephalogr Clin Neurophysiol Suppl. 1999;52:21-41.

2. Goodwin JE. The significance of alpha variants in the EEG, and their relationship to an epileptiform syndrome. Am J Psychiatry. 1947 Dec;104(6):369-79.

3. Aird RB, Gastaut Y. Occipital and posterior electroencephalographic rhythms. Electroencephalogr Clin Neurophysiol. 1959 Nov; 11: 637-56.

4. Westmoreland BF, Klass DW. Unusual EEG patterns. J Clin Neurophysiol. 1990 Apr;7(2):209-28.

5. Westmoreland BF, Klass DW. A distinctive rhythmic EEG discharge of adults. Electroencephalogr Clin Neurophysiol. 1981;51:186-91. 